\title{
Elucidating the atomistic mechanisms driving self-diffusion of amorphous Si during annealing
}

\author{
Iván Santos, " Luis A. Marqués, and Lourdes Pelaz \\ Departamento de Electricidad y Electrónica, Universidad de Valladolid, E.T.S.I. de Telecomunicación, Paseo Belén 15, \\ E-47011 Valladolid, Spain \\ Luciano Colombo \\ Dipartimento di Fisica, Università degli Studi di Cagliari, Cittadella Universitaria, I-09042 Monserrato (CA), Italy
} (Received 23 November 2010; revised manuscript received 8 February 2011; published 14 April 2011)

\begin{abstract}
We have analyzed the atomic rearrangements underlying self-diffusion in amorphous Si during annealing using tight-binding molecular dynamics simulations. Two types of amorphous samples with different structural features were used to analyze the influence of coordination defects. We have identified several types of atomic rearrangement mechanisms, and we have obtained an effective migration energy of around $1 \mathrm{eV}$. We found similar migration energies for both types of samples, but higher diffusivities in the one with a higher initial percentage of coordination defects.
\end{abstract}

DOI: 10.1103/PhysRevB.83.153201

PACS number(s): 07.05.Tp, 66.30.-h, 81.05.Gc

Despite the renewed interest in amorphous $\mathrm{Si}(a-\mathrm{Si})$ for optoelectronic and photovoltaic applications, ${ }^{1}$ the atomicscale understanding of its microstructural evolution during processing (an issue of large technological impact) is still a matter of debate. In particular, it has been found that impurity diffusion is greatly affected by modifications in the $a$-Si structure. ${ }^{2,3}$ These modifications mainly occur during annealing, and they are directly related to microscopic changes of the amorphous matrix. ${ }^{4}$ Uncontrolled diffusion during the fabrication process results in the degradation of the final performance of devices. A better understanding of diffusion at the atomic level will help to overcome these negative effects.

In this Brief Report, we have analyzed the atomic rearrangements underlying self-diffusion in $a$-Si during annealing. We have used tight-binding molecular dynamics (TBMD) simulations within an $\mathrm{sp}^{3}$ orthogonal tight-binding approach, ${ }^{5,6}$ and the Hamiltonian representation proposed by Kwon et al. ${ }^{7}$ This representation has been successfully used for studying diffusion phenomena in crystalline $\mathrm{Si}$ (Refs. 8-10) $(c$-Si) and the properties of $a-\mathrm{Si}^{11-13}$ We have considered two models of $a-\mathrm{Si}$, obtained by rather different computer simulations. On the one hand, $a$-Si was obtained by melting $c$-Si, and quenching the resulting liquid at a rate of $10^{13} \mathrm{~K} / \mathrm{s}$, which lies within the typical cooling rates used in molecular dynamics simulations. ${ }^{14}$ On the other hand, $a$-Si was obtained using the algorithm developed by Wooten, Winer, and Weaire (WWW). ${ }^{15}$ We considered three quenched and two WWW cubic cells with 216 atoms. The structural differences between these two types of $a$-Si simulation cells are well known: while the WWW algorithm generates a perfectly fourfold coordinated $a-\mathrm{Si}_{1}{ }^{15}$ quenched samples have an appreciable occurrence of non fourfold coordinated atoms that depends on the quench rate. ${ }^{14}$ In our case, we had $\sim 80 \%$ and $\sim 20 \%$ of fourfold and fivefold coordinated atoms, respectively, using a bond cutoff for determining the local atomic coordination as long as $2.8 \AA$. The aim of considering two different models of $a$-Si is to investigate the possible role of coordination defects on the microstructure evolution during annealing. Generated samples were rapidly heated at the desired temperature and stabilized during 5 ps before analyzing the dynamics of the system. Then, we performed constant temperature and volume simulations for total times ranging from 1.5 to $4 \mathrm{~ns}$. The temperature range was chosen between 900 and $1300 \mathrm{~K}$, since for higher temperatures, $a$-Si transforms into liquid. ${ }^{11}$ We performed velocity rescalings every $10^{3}$ time steps to keep the temperature of the cell constant. Equations of motion were integrated using the velocity-Verlet algorithm ${ }^{16}$ with a time step of 1.5 fs. Periodic boundary conditions were applied in all directions.

To analyze self-diffusion, we evaluated the atomic meansquared displacement (MSD) as

$$
\operatorname{MSD}(t)=\sum_{i=1}^{N} \frac{\left|\mathbf{r}_{i}(t)-\mathbf{r}_{i}(0)\right|^{2}}{N},
$$

with $N$ the number of atoms in the simulation cell, and $\mathbf{r}_{i}(t)$ the position of atom $i$ at time $t$. A representative plot of the time evolution of the MSD in the case of quenched $a-\mathrm{Si}$ at several temperatures is shown in Fig. 1. At temperatures lower than $1000 \mathrm{~K}$, the atomic MSD slowly increases with time by means of small steps separated by long plateaus. As temperature increases, the steps are higher and plateaus become shorter. Bearing in mind that abrupt changes in the MSD have been correlated to transitions among different configurations of the $\mathrm{Si}$ self-interstitial in $c-\mathrm{Si}^{17}$ we paid attention to atomic trajectories when abrupt changes in the MSD occurred in some of the simulations performed. We used the fast Fourier transformation (FFT) to filter out thermal vibrations for identifying relevant atomic movements in our simulations. The FFT is applied to atomic trajectories to obtain the frequency spectrum. Since frequencies associated to lattice vibrations are much higher than those associated to atomic rearrangements, an inverse FFT in which high frequencies are neglected is performed to obtain filtered atomic trajectories and keep only those events that really induce microstructural changes. Contrary to atomic diffusion in $c$-Si, where the number of stable and saddle configurations is limited, the underlying amorphous network in $a$-Si varies as diffusion events take place. The initial atomic configuration before 


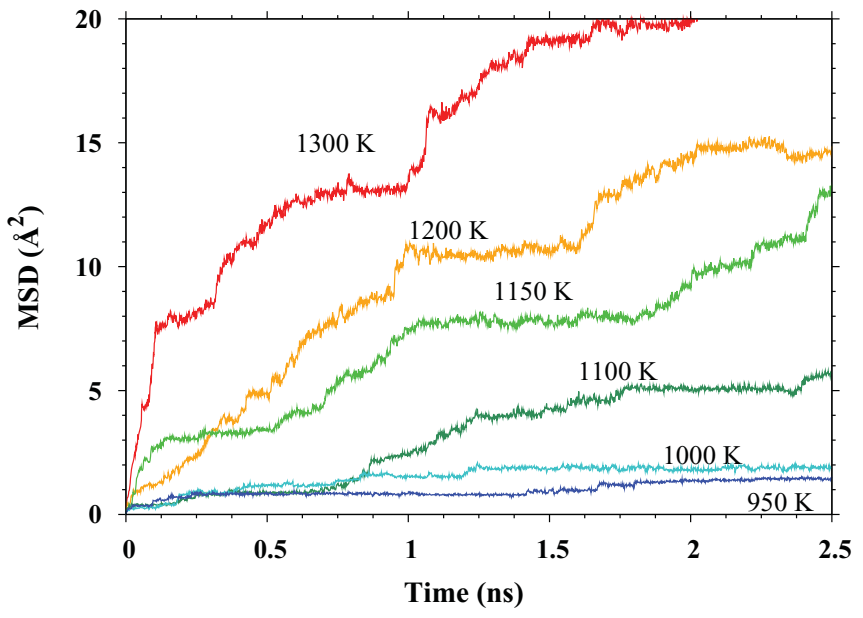

FIG. 1. (Color online) Representative plot for the MSD in the case of quenched $a-\mathrm{Si}$ as a function of time for different temperatures.

diffusion events differs one from another, and so it does the path followed by the atoms that move. This fact results in a distribution of energy barriers, as reported in theoretical studies of the configurational energy landscape in $a$-Si. ${ }^{18}$ Nevertheless, we could identify several common features in the observed atomic rearrangements that allowed us to classify them. By analyzing the broken and newly formed bonds and the atomic displacements, we have identified five different types of basic mechanisms, namely: bond break (BB), bond switch (BS), frustrated bond switch (FBS), kick-out (KO), and vacancy-like (VL). The rearrangements shown in Fig. 2 are representative examples of the prototypical mechanisms identified. The features that define these mechanisms, which will be described in the following, were used to classify the rearrangements that took place during the simulations of annealing.

The BB only involves one atom (A) in the rearrangement. When one of its bonds is broken (bond $\mathrm{A}-\mathrm{B}$ ), the influence of the rest of its neighbors causes the atom $\mathrm{A}$ to move in the opposite direction to the broken bond. It forms a new bond with another atom $(\mathrm{C})$ which was initially at a second-neighbor distance. Atomistic models have proposed the $\mathrm{BB}$ as a possible mechanism for the creation of light-induced defects in hydrogenated $a-\mathrm{Si}^{19}$ The BS consists of the interchange of two neighbors $(C, D)$ between two neighboring atoms $(\mathrm{A}, \mathrm{B})$ : the bonds $\mathrm{A}-\mathrm{D}$ and $\mathrm{B}-\mathrm{C}$ are switched to become $\mathrm{A}-\mathrm{C}$ and $\mathrm{B}-\mathrm{D}$. While the $\mathrm{BS}$ mechanism was only conjecture to date, ${ }^{18}$ in the present study, by analyzing the TBMD trajectories, we provide evidence that it indeed occurs and is responsible for large local rearrangements. Furthermore, our results also indicate that $\mathrm{BS}$ in $a$-Si can be produced by thermal atomic motions in addition to light illumination, as proposed by Wagner et al. ${ }^{20}$ The BS has a special relevance in both $c$-Si and $a$-Si. The so-called IV pair is a point defect in $c-\mathrm{Si}$ that can be generated and recombined through a BS. ${ }^{8,21}$ This defect plays an important role in describing the amorphization and the recrystallization processes of $\mathrm{Si}^{21}$ In addition, the so-called concerted-exchange diffusion mechanism in $c$-Si can be regarded as a combination of three consecutive BSs involving the same pair of atoms. ${ }^{22}$ Moreover, the BS is the fundamental movement within the WWW bond network for the generation of $a$-Si from $c$-Si.

Due to rearrangements induced in the surroundings, sometimes the neighbor interchange is not totally completed in the BS event. This feature is manifested through the identification of a FBS rearrangement, which is shown in Fig. 2. The bonds $\mathrm{A}-\mathrm{D}$ and $\mathrm{B}-\mathrm{C}$ break as it occurs in the $\mathrm{BS}$, and the bond $\mathrm{B}-\mathrm{D}$ is formed. However, atom $\mathrm{A}$ does not bond to atom $\mathrm{C}$ but rather to atom $\mathrm{E}$, which is a second neighbor of atom $\mathrm{B}$. Although at the end of the movement the newly formed bonds are $\mathrm{A}-\mathrm{E}$ and $\mathrm{B}-\mathrm{D}$, this type of rearrangement has a BS nature. During the KO event, one atom (A) displaces another one (B) from its position. As a consequence of this rearrangement, atom $\mathrm{C}$ is also displaced in this particular case. This kind of movement is similar to that occurring during the diffusion of $\mathrm{Si}$ self-interstitials in $c$-Si. ${ }^{8,17,23}$ Finally, in the VL, only one atom (A) moves as in the BB, but in the VL, the distance traveled is longer. In the case of the VL, atom A seeks open spaces in the network, and changes most of its neighbors during the displacement. This movement resembles the vacancy diffusion mechanism in $c$-Si where an atom moves toward the empty

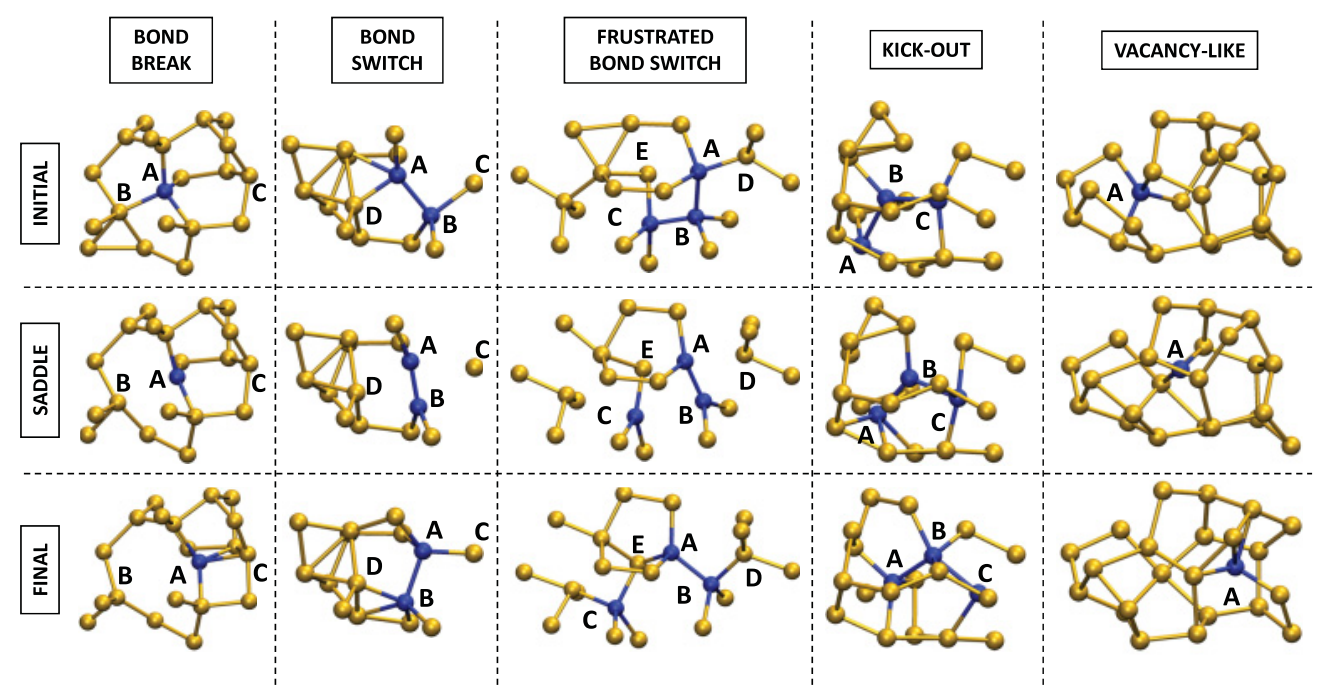

FIG. 2. (Color online) Snapshots of representative atomic movements in the observed rearrangement mechanisms. See text for details. 


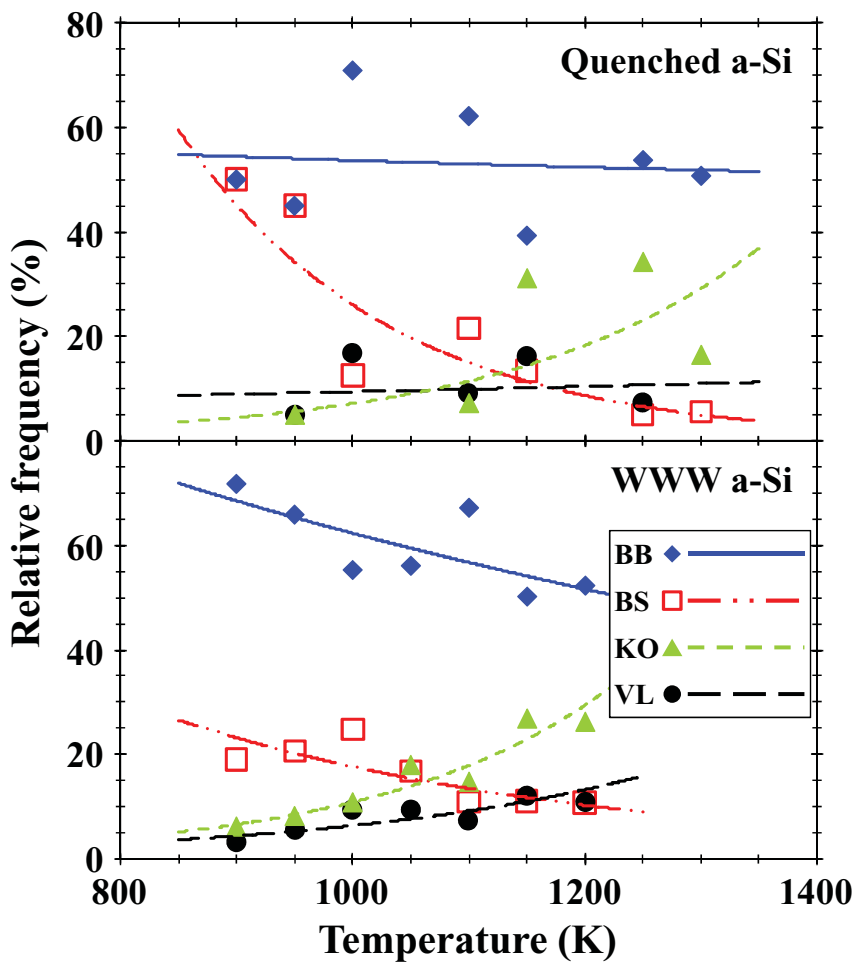

FIG. 3. (Color online) Statistics of the observed basic diffusion mechanisms. Lines are to guide the eye.

space associated with a vacancy. ${ }^{24}$ It is worth noting that, although the BB and the BS events found here agree with the observations of previous theoretical static studies, ${ }^{18}$ neither the $\mathrm{KO}$ nor the VL events were reported.

In order to evaluate the relevance of the observed mechanisms, we have represented in Fig. 3 the relative frequency of a given event as a function of temperature. Since sometimes it is difficult to distinguish between the FBS and the BS, we have gathered FBS events within the BS group. We have found that the BB rearrangement is the more frequent event in both samples at the analyzed temperatures. The BS has an important contribution to atomic rearrangements at low temperatures, especially in quenched $a-\mathrm{Si}$, but its relevance diminishes as temperature increases. The relative frequency of the $\mathrm{KO}$ events increase with temperature, while in the case of VL, the increase is less. In general, it can be inferred from Fig. 3 that coordination defects favor BS events, while $\mathrm{KO}$ and VL events take place when the temperature is high enough. All these rearrangements caused the steps in the MSD observed in Fig. 1. Small steps at low temperatures are associated with isolated rearrangements involving small displacements of few atoms. As temperature increases, the higher steps are related to an increment of the complexity of rearrangements involving a larger number of atoms, which can be regarded as a succession of several "basic rearrangements," with BB the usual triggering event.

We have also calculated the atomic diffusivity $d(T)$ from the MSD at different temperatures using the Einstein relation

$$
d(T)=\lim _{t \rightarrow \infty} \frac{\operatorname{MSD}(t)}{6 t} .
$$

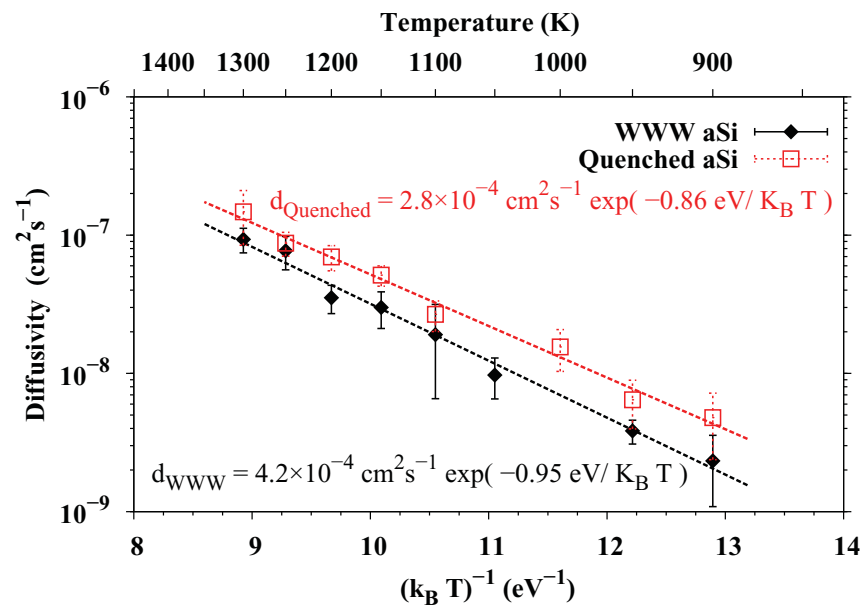

FIG. 4. (Color online) Average atomic diffusivities at each temperature of the samples considered. Straight lines and equations show the result of the Arrhenius data fit.

In Fig. 4 we represent the average diffusivity at each temperature for the samples considered. Quenched samples have higher atomic diffusivities than WWW ones. This may be due to their higher initial structural distortion, which appears to enhance atomic rearrangement events. Data can be described with an Arrhenius behavior within the temperature range analyzed as

$$
d(T)=d_{0} \exp \left(-\frac{E_{m}}{k_{B} T}\right)
$$

where $d_{0}$ is the diffusivity prefactor, $E_{m}$ is the effective migration energy for self-diffusion in $a$-Si, $k_{B}$ is the Boltzmann constant, and $T$ is the temperature. The result of the Arrhenius fits is shown in Fig. 4. The obtained effective migration energies are $0.86 \mathrm{eV}$ and $0.95 \mathrm{eV}$ for quenched and WWW $a$-Si, respectively. These energies are of the order of magnitude of the migration energies of point defects in $c$-Si. ${ }^{8,9,17,23,24} \mathrm{In}$ addition, as we described above, we also observed analogous local atomic rearrangements in both $c$-Si and $a$-Si. These facts are consequences of the similar short-range atomic environment that both phases of silicon share.

The evaluated migration energy captures the statistical average behavior of different atomic rearrangements in a dynamical system. Calorimetric ${ }^{4}$ and conductivity ${ }^{25}$ measures on $a$-Si have revealed the existence of activation energy barriers ranging from $\sim 0.25 \mathrm{eV}$ to $\sim 2.7 \mathrm{eV}$. Theoretical static studies of the configurational energy landscape in $a$-Si have shown that there exists a distribution of energy barriers for the saddle point and for the energy difference between initial and final configurations. ${ }^{18}$ In spite of this complexity, it has been demonstrated that in amorphous materials, the combined effect of both distributions can result in an Arrhenius diffusion behavior. ${ }^{26,27}$

In conclusion, we have studied, from an atomistic point of view, the microstructure evolution of $a$-Si during annealing using TBMD simulations. The evaluation of the MSD together with the analysis of atomic trajectories enabled us to make a synopsis of the type and relevance of rearrangement events 
occurring during annealing. Despite the variety of mechanisms found, an effective migration energy of $\sim 1 \mathrm{eV}$ was obtained from the Arrhenius plot of the atomic diffusivities that encompasses their average behavior in self-diffusion in $a$-Si. This energy could be used in Monte Carlo simulations of the microstructure evolution of $a$-Si in order to access time scales that go beyond molecular dynamics simulations.
The authors thank L. Bagolini for providing the WWW $a$-Si cells. This work was partially funded by HPC-EUROPA2 under Project No. 228398 with the support of the European Commission Capacities Area - Research Infrastructures Initiative, Spanish DGI under Project No. TEC2008-06069, and Junta de Castilla y León under Project No. VA011A09 (IS, LAM, LP). *ivasan@tel.uva.es

${ }^{1}$ L. Khriachtchev, Silicon Nanophotonics (World Scientific, Singapore, 2008).

${ }^{2}$ S. Coffa, J. M. Poate, D. C. Jacobson, W. Frank, and W. Gustin, Phys. Rev. B 45, 8355 (1992).

${ }^{3}$ S. Mirabella, D. DeSalvador, E. Bruno, E. Napolitani, E. F. Pecora,

S. Boninelli, and F. Priolo, Phys. Rev. Lett. 100, 155901 (2008).

${ }^{4}$ S. Roorda, W. C. Sinke, J. M. Poate, D. C. Jacobson, S. Dierker, B. S. Dennis, D. J. Eaglesham, F. Spaepen, and P. Fuoss, Phys. Rev. B 44, 3702 (1991).

${ }^{5}$ L. Colombo and M. Rosati, Comput. Phys. Commun. 128, 108 (2000).

${ }^{6}$ L. Colombo, Comput. Mater. Sci. 12, 278 (1998).

${ }^{7}$ I. Kwon, R. Biswas, C. Z. Wang, K. M. Ho, and C. M. Soukoulis, Phys. Rev. B 49, 7242 (1994).

${ }^{8}$ M. Tang, L. Colombo, J. Zhu, and T. Diaz de la Rubia, Phys. Rev. B 55, 14279 (1997).

${ }^{9}$ L. Colombo, Annu. Rev. Mater. Res. 32, 271 (2002).

${ }^{10}$ M. Cogoni, B. P. Uberuaga, A. F. Voter, and L. Colombo, Phys. Rev. B 71, 121203 (2005).

${ }^{11}$ V. Rosato and M. Celino, J. Appl. Phys. 86, 6826 (1999).

${ }^{12}$ P. Biswas, Phys. Rev. B 65, 125208 (2002).

${ }^{13}$ D. A. Drabold, Y. Li, B. Cai, and M. Zhang, Phys. Rev. B 83, 045201 (2011).
${ }^{14}$ M. Ishimaru, S. Munetoh, and T. Motooka, Phys. Rev. B 56, 15133 (1997).

${ }^{15}$ F. Wooten, K. Winer, and D. Weaire, Phys. Rev. Lett. 54, 1392 (1985).

${ }^{16}$ W. C. Swope et al., J. Chem. Phys. 76, 637 (1982).

${ }^{17}$ L. A. Marqués, L. Pelaz, P. Castrillo, and J. Barbolla, Phys. Rev. B 71, 085204 (2005).

${ }^{18}$ F. Valiquette and N. Mousseau, Phys. Rev. B 68, 125209 (2003).

${ }^{19}$ M. Stutzmann, W. B. Jackson, and C. C. Tsai, Phys. Rev. B 32, 23 (1985).

${ }^{20}$ L. K. Wagner and J. C. Grossman, Phys. Rev. Lett. 101, 265501 (2008).

${ }^{21}$ L. A. Marqués, L. Pelaz, M. Aboy, L. Enriquez, and J. Barbolla,Phys. Rev. Lett. 91, 135504 (2003).

${ }^{22}$ K. C. Pandey, Phys. Rev. Lett. 57, 2287 (1986).

${ }^{23}$ M. Posselt, F. Gao, and H. Bracht, Phys. Rev. B 78, 035208 (2008).

${ }^{24}$ P. M. Fahey, P. B. Griffin, and J. D. Plummer, Rev. Mod. Phys. 61, 290 (1989).

${ }^{25}$ J. H. Shin and H. A. Atwater, Phys. Rev. B 48, 5964 (1993).

${ }^{26}$ Y. Limoge and J. L. Bocquet, Phys. Rev. Lett. 65, 60 (1990).

${ }^{27}$ K. Mussawisade, T. Wichmann, and K. W. Kehr, J. Phys. Condens. Matter 9, 1181 (1997). 